\title{
The incidence and survival of cervical, ovarian, and endometrial cancer in Korea, 1999-2017: Korea Central Cancer Registry
}

\author{
Hyeong In Ha, MD ${ }^{1,2}$, Ha Kyun Chang, MD, PhD³, Soo Jin Park, MD4, Jiwon Lim, MS ${ }^{5}$, Young-Joo Won, PhD ${ }^{5,6}$, \\ Myong Cheol Lim, MD, PhD $2,6,7$

\begin{abstract}
'Department of Obstetrics and Gynecology, Pusan National University Yangsan Hospital, Pusan National University School of Medicine, Yangsan; ${ }^{2}$ Center for Gynecologic Cancer, Research Institute and Hospital, National Cancer Center, Goyang; ${ }^{3}$ Department of Obstetrics and Gynecology, Korea University Ansan Hospital, Ansan; ${ }^{4}$ Department of Obstetrics and Gynecology, Seoul National University College of Medicine, Seoul; ${ }^{5}$ Division of Cancer Registration and Surveillance, National Cancer Control Institute, National Cancer Center, ${ }^{6}$ Department of Cancer Control and Population Health, National Cancer Center Graduate School of Cancer Science and Policy, ${ }^{7}$ Division of Tumor Immunology, Research Institute, National Cancer Center, Goyang, Korea
\end{abstract}

\section{Objective}

The three major gynecologic cancers are cervical, endometrial, and ovarian cancer. This study aimed to describe the 19-year trends and survival rates in cervical, endometrial, and ovarian cancer in a Korean female population.

\section{Methods}

We searched the Korea Central Cancer Registry to identify patients with gynecologic cancer between 1999 and 2017. Age-standardized rates and annual percent changes were calculated. The relative survival rate (RSR) was reported by histology, age, and stage for each gynecological cancer.

\section{Results}

The total number of cervical, endometrial, primary peritoneal, ovarian epithelial, fallopian tube (POFT) cancer was 134,863, with the number of cases increasing every year: 6,077 in 1999 to 8,011 in 2017. The incidence of cervical cancer has decreased; however, that of POFT and endometrial cancer has increased. The 5-year RSR of cervical, POFT, and endometrial cancer was reported to be $80.8 \%, 61.4 \%$, and $88.1 \%$, respectively. In the case of cervical cancer, squamous cell carcinoma showed better survival than other histology ( $82.8 \%$ vs. $73.5 \%)$. Furthermore, in the case of endometrial cancer, endometrioid histology had substantially better 5-year RSR than the others (93.2\% vs. $76.5 \%)$. Contrastingly, in the case of ovarian cancer, serous carcinoma had worse 5-year RSR than other types of histology.

\section{Conclusion}

The incidence rates for gynecologic cancers increased from 2005 to 2017, with an annual increase of 2.76 per year until 2017. Endometrial cancer had the highest RSR, while ovarian cancer had the lowest. Active cancer screening and the introduction of effective treatments might have contributed to the improved RSRs of gynecologic cancers.

Keywords: Ovarian neoplasms; Endometrial neoplasms; Uterine cervical neoplasms; Survival

Received: 2021.04.05. Revised: 2021.06.07. Accepted: 2021.08.01.

Corresponding author: Young-Joo Won, PhD

Division of Cancer Registration and Surveillance, National Cancer

Control Institute, National Cancer Center, 323 Ilsan-ro, Ilsandong-

gu, Goyang 10408, Korea

E-mail: astra67@ncc.re.kr

https://orcid.org/0000-0001-9861-6740
Myong Cheol Lim, MD, PhD

Center for Gynecologic Cancer, Research Institute and Hospital,

National Cancer Center, 323 Ilsan-ro, Ilsandong-gu, Goyang

10408, Korea

E-mail: gynlim@gmail.com

https://orcid.org/0000-0001-8964-7158

Articles published in Obstet Gynecol Sci are open-access, distributed under the terms of the Creative Commons Attribution Non-Commercial License (http://creativecommons.org/ licenses/by-nc/3.0/) which permits unrestricted non-commercial use, distribution, and reproduction in any medium, provided the original work is properly cited. 


\section{Obstetrics \& Gynecology Science}

Hyeong In Ha, et al. The incidence and survival of gynecologic cancer in Korea in 1999-2017

\section{Introduction}

Cancer is an important public health concern and the leading cause of death in Korea in 2017. Indeed, cancers of the cervix and ovary are the eighth and ninth most common causes of death from cancer in Korea, respectively. Furthermore, cervical, ovarian, and endometrial cancers are responsible for $6.7 \%$ of all cancer deaths in Korea during the same period [1]. Cervical, endometrial, and ovarian cancers account for the largest proportion of cancers in the female genital organs. These cancers occur in adjacent organs, whereas their incidence varies in different patterns in the Korean female popu-

Table 1. Trends in gynecologic cancer incidence rate by cancer type, 1999-2017

\begin{tabular}{|c|c|c|c|c|c|c|c|c|c|}
\hline \multirow{2}{*}{\multicolumn{2}{|c|}{ Year }} & \multicolumn{2}{|c|}{ Total } & \multicolumn{2}{|c|}{ Cervix } & \multicolumn{2}{|c|}{ POFT } & \multicolumn{2}{|c|}{ Endometrium } \\
\hline & & Cases & ASR & Cases & ASR & Cases & ASR & Cases & ASR \\
\hline \multicolumn{2}{|l|}{1999} & 6,077 & 22.5 & 4,487 & 16.4 & 967 & 3.7 & 623 & 2.4 \\
\hline \multicolumn{2}{|l|}{2000} & 5,972 & 21.4 & 4,335 & 15.4 & 998 & 3.7 & 639 & 2.4 \\
\hline \multicolumn{2}{|l|}{2001} & 6,391 & 22.3 & 4,646 & 16.0 & 1,016 & 3.7 & 729 & 2.6 \\
\hline \multicolumn{2}{|l|}{2002} & 6,393 & 21.8 & 4,493 & 15.1 & 1,082 & 3.8 & 818 & 2.9 \\
\hline \multicolumn{2}{|l|}{2003} & 6,452 & 21.4 & 4,379 & 14.2 & 1,149 & 4.0 & 924 & 3.2 \\
\hline \multicolumn{2}{|l|}{2004} & 6,268 & 20.2 & 4,144 & 13.2 & 1,202 & 4.0 & 922 & 3.1 \\
\hline \multicolumn{2}{|l|}{2005} & 6,442 & 20.2 & 4,037 & 12.5 & 1,312 & 4.3 & 1,093 & 3.5 \\
\hline \multicolumn{2}{|l|}{2006} & 6,600 & 20.2 & 4,064 & 12.3 & 1,378 & 4.3 & 1,158 & 3.6 \\
\hline \multicolumn{2}{|l|}{2007} & 6,643 & 19.9 & 3,777 & 11.1 & 1,576 & 4.8 & 1,290 & 3.9 \\
\hline \multicolumn{2}{|l|}{2008} & 7,048 & 20.5 & 4,022 & 11.6 & 1,548 & 4.6 & 1,478 & 4.4 \\
\hline \multicolumn{2}{|l|}{2009} & 7,060 & 20.0 & 3,832 & 10.7 & 1,592 & 4.5 & 1,636 & 4.8 \\
\hline \multicolumn{2}{|l|}{2010} & 7,412 & 20.7 & 4,002 & 11.0 & 1,753 & 5.0 & 1,657 & 4.7 \\
\hline \multicolumn{2}{|l|}{2011} & 7,375 & 20.2 & 3,804 & 10.3 & 1,773 & 4.9 & 1,798 & 4.9 \\
\hline \multicolumn{2}{|l|}{2012} & 7,462 & 20.0 & 3,671 & 9.8 & 1,949 & 5.2 & 1,842 & 4.9 \\
\hline \multicolumn{2}{|l|}{2013} & 7,761 & 20.3 & 3,700 & 9.7 & 1,996 & 5.2 & 2,065 & 5.4 \\
\hline \multicolumn{2}{|l|}{2014} & 7,841 & 20.3 & 3,569 & 9.2 & 2,174 & 5.6 & 2,098 & 5.4 \\
\hline \multicolumn{2}{|l|}{2015} & 8,142 & 20.6 & 3,631 & 9.2 & 2,225 & 5.6 & 2,286 & 5.8 \\
\hline \multicolumn{2}{|l|}{2016} & 8,713 & 21.8 & 3,600 & 9.2 & 2,473 & 6.1 & 2,640 & 6.5 \\
\hline \multicolumn{2}{|l|}{2017} & 8,811 & 21.7 & 3,469 & 8.7 & 2,505 & 6.1 & 2,837 & 7.0 \\
\hline \multicolumn{2}{|c|}{$1999-2017$} & 134,863 & 20.7 & 75,662 & 11.5 & 30,668 & 4.8 & 28,533 & 4.4 \\
\hline \multirow[t]{4}{*}{ Trend 1} & Range (yr) & \multicolumn{2}{|c|}{$1999-2005$} & & & & & & \\
\hline & APC & & & & & & & & \\
\hline & $95 \% \mathrm{Cl}$ & $(-2.8$ & .65) & $(-5$. & 68) & & & $(6.2$ & \\
\hline & $P$-value & & & & & & & & \\
\hline Trend 2 & Range (yr) & 200 & & & & & & & \\
\hline & APC & & & & & & & & \\
\hline & $95 \% \mathrm{Cl}$ & $(-0)$. & .66) & $(-3$. & .13) & & & $(0.6$ & \\
\hline & $P$-value & & & & & & & & \\
\hline Trend 3 & Range (yr) & 20 & & & & & & 20 & \\
\hline & APC & & & & & & & & \\
\hline & $95 \% \mathrm{Cl}$ & $(-0.2$ & .81) & & & & & $(4.9$ & \\
\hline & $P$-value & & & & & & & & \\
\hline
\end{tabular}

POFT, primary peritoneal, ovarian epithelial, fallopian tube; ASR, age-standardized rates; APC, annual percent changes; $\mathrm{Cl}$, confidence interval. 


\section{Obstetrics \& Gynecology Science}

Vol. 64, No. 5, 2021

lation. To investigate the change in the trends and survival of gynecological cancer, several researchers have analyzed the incidence of gynecological cancer in Korean women using data from the Korea Central Cancer Registry (KCCR) at intervals of several years [2-4]. In recent decades, the incidence of gynecological cancer has changed dynamically, becoming similar to patterns found in Western countries. Starting in 2016, the number of new cases was in the order of cervical, ovarian, and uterine corpus cancer. However, from 2016 onwards, the number of new cases was in the order of cervical, uterine corpus, and ovarian cancer [5]. This study aimed to describe the 19-year trends and survival rates in cervical, endometrial, and ovarian cancer in a Korean female population.

\section{Materials and methods}

This study used data from patients enrolled in the KCCR, which collects information regarding approximately 98\% [1] of cancer cases in Korea since 1999. According to the cancer registration guidelines, only the date of diagnosis, diagnosis route, primary site, laterality, histology, degree of differentiation, diagnostic method, and treatment modality were recorded.
We searched the KCCR to identify all patients with cervical, ovarian, and endometrial cancer diagnosed between 1999 and 2017. We analyzed primary peritoneal, epithelial ovarian, and fallopian tube (POFT) cancer together with the ovary because they were treated as one entity. Cervical cancer (C53), ovarian cancer (C48, C56, and C57), and endometrial cancer (C54.1) were defined based on the International Classification of Diseases, 10th revision [6].

Age-standardized rates (ASRs) were calculated in patients diagnosed with gynecologic cancer using Segi's world standard population [7] and were expressed per 100,000 women. The trends in annual ASRs were estimated by calculating annual percent changes (APCs) using the following

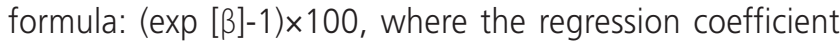
$(\beta)$ was calculated by a linear regression of log-transformed age-standardized incidence rates for the period from 1999 to 2017. The relative survival rates (RSRs), defined as the ratio of observed survival of cancer patients to expected survival in the general population, were analyzed according to age at diagnosis ( $<50$ and $\geq 50$ years), the Surveillance, Epidemiology, and End Results (SEER) summary stage [8], and histologic type. RSRs were adjusted for the effects of other causes of death using the standard population life table provided by Statistics Korea (http://kosis.kr) and were estimated using the Ederer II method with some minor corrections, based on an

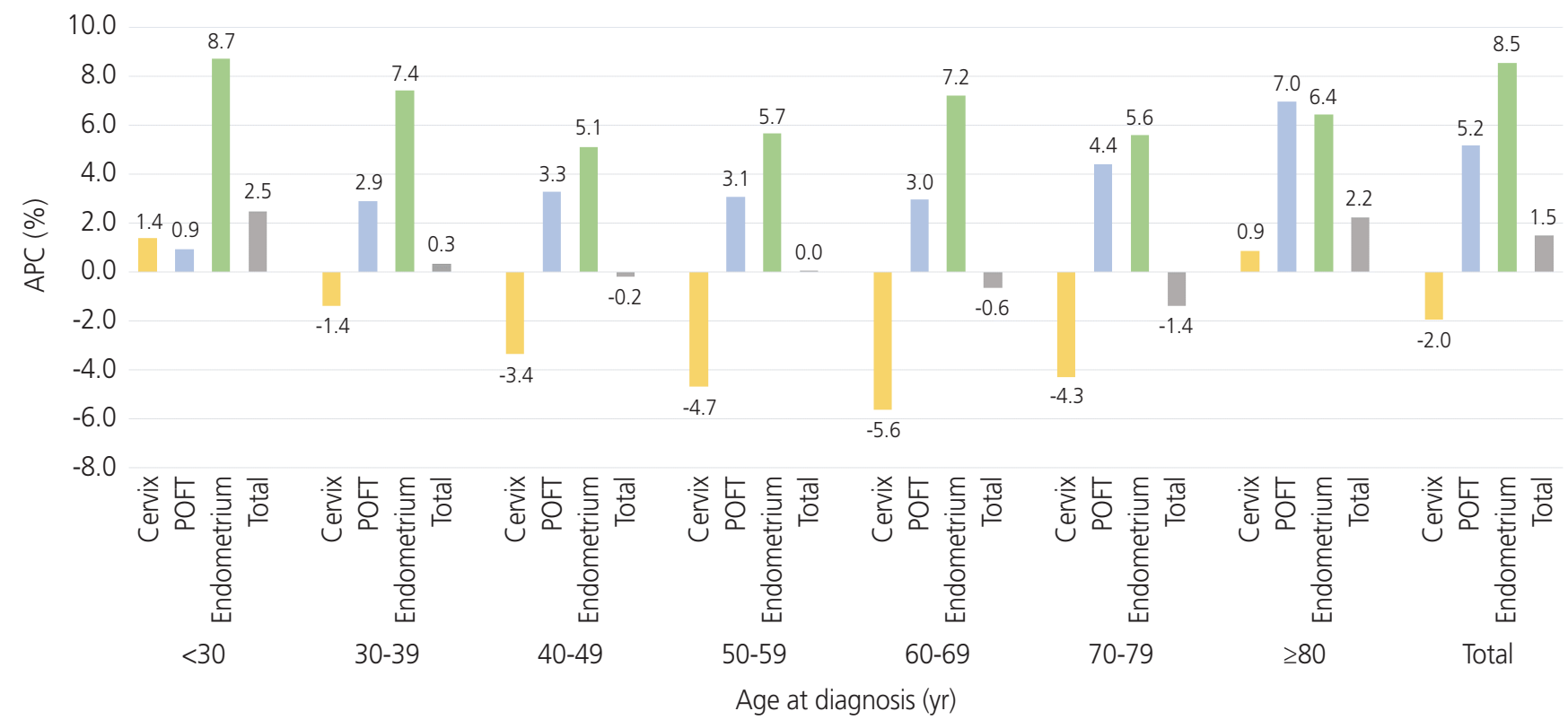

Fig. 1. Annual percent changes (APC) in the incidence by age group and cancer type, 1999-2017. POFT, primary peritoneal, ovarian epithelial, fallopian tube. 


\section{Obstetrics \& Gynecology Science}

Hyeong In Ha, et al. The incidence and survival of gynecologic cancer in Korea in 1999-2017

algorithm written in SAS by Paul Dickman [9]. All statistical tests were considered statistically significant at $P$-value $<0.05$. Analyses of incidence and survival rates were performed using SAS (version 9.4; SAS Institute Inc., Cary, NC, USA), while the analysis of trends was conducted using Join Point 4.7.0.0 (National Cancer Institute, Bethesda, MD, USA).

This study was approved by the Institutional Review Board of the National Cancer Center, Korea (NCC2020-0176). The requirement for informed consent was waived due to the deidentified nature of secondarily analyzed data.

\section{Results}

The absolute overall incidence of cervical, POFT, and endometrial cancer has increased from 6,077 in 1999 to 8,811

A

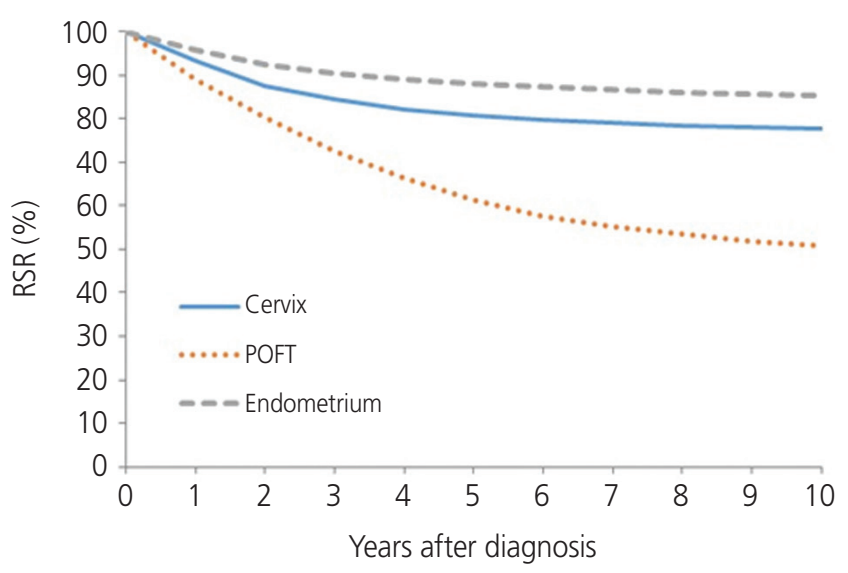

C

POFT

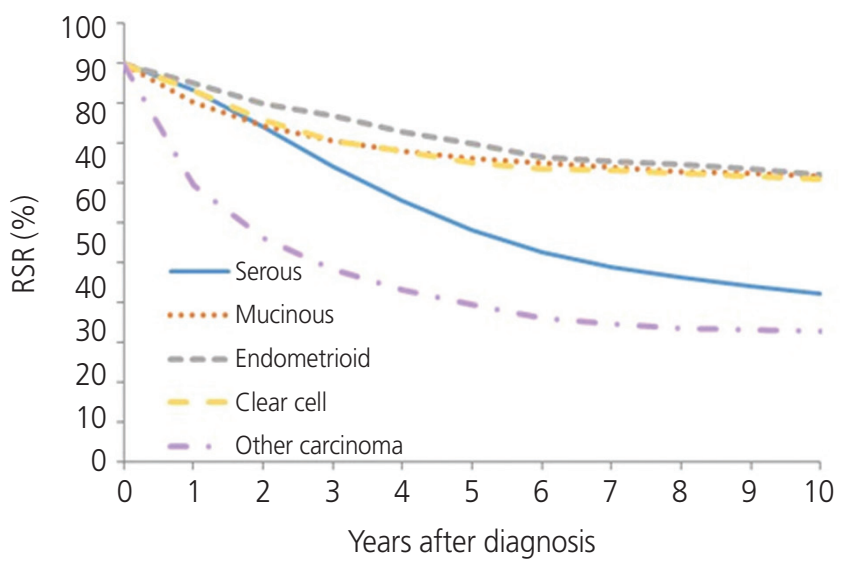

in 2017 (Table 1). Each incidence rate in gynecologic cancer described by histology, age at diagnosis, and SEER stages was organized in Supplementary Table 1-3. However, the absolute number of cervical cancer cases decreased from 4,487 in 1999 to 3,469 in 2017. The overall and site-specific ASRs and APCs are listed in Table 1. The ASR for gynecologic cancer decreased from 22.5 per 100,000 in 1999 to 21.7 in 2017. The APCs during the same period were $-1.76 \%$ (95\% confidence interval $[\mathrm{Cl}]=-2.86,-0.65)$ between 1999 and $2005,-0.03 \%(95 \% \mathrm{Cl}=-0.71,0.66)$ between 2005 and 2014, and 2.76\% (95\% Cl=-0.20, 5.81) between 2014 and 2017. The incidence of cervical cancer decreased from an ASR of 16.4 in 1999 to 8.7 in 2017. The APC during the same period was $-4.56 \%(95 \% \mathrm{Cl}=-5.43,-3.68)$ between 1999 and 2007 , and $-2.82 \%(95 \% \mathrm{Cl}=-3.51,-2.13)$ between 2007 and 2017. Fig. 1 shows the overall APCs for the 3

B

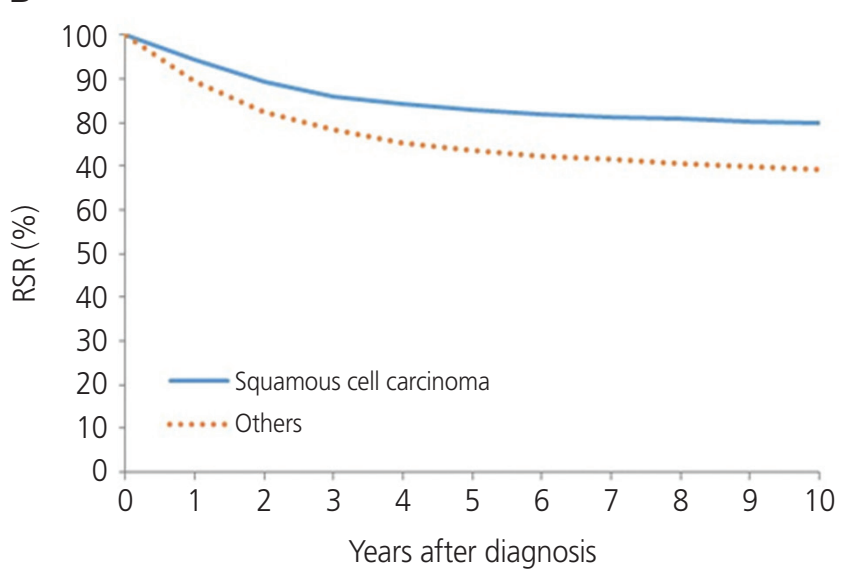

D

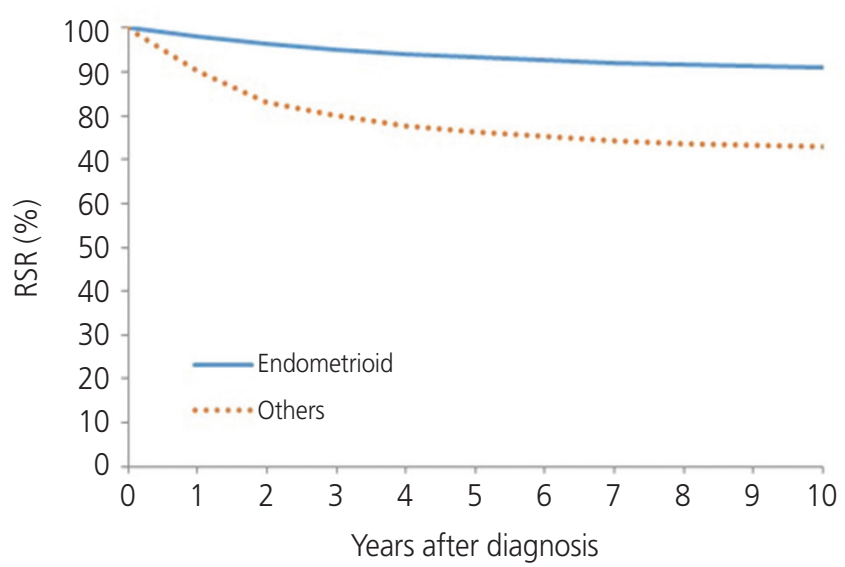

Fig. 2. Relative survival rate (RSR) by cancer type and histologic group, 1999-2017 (A-D). POFT, primary peritoneal, ovarian epithelial, fallopian tube. 


\section{Obstetrics \& Gynecology Science}

Vol. 64, No. 5, 2021

major gynecologic cancers according to age group and cancer type between 1999 and 2017. Analyzing by age group, the incidence of cervical cancer has been decreasing in all age groups except in those aged younger than 30 years and 80 years or older. Especially between the 30-39 years and 70-79 years age groups, cervical cancer showed the most rapidly decreasing incidence during this observation period (APC, $-3.4 \%,-4.7 \%,-5.6 \%$, and $-4.3 \%$, respectively). However, cervical cancer has the widest age distribution (Supplementary Table 4). Fig. 2A shows that the 5-year RSR of cervical, POFT, and endometrial cancer was reported to be $80.8 \%, 61.4 \%$, and $88.1 \%$, respectively $(P<0.0001)$.

The squamous cell carcinoma histology of cervical cancer showed better survival than non-squamous cell carcinoma histology in terms of 5-year RSR $(82.8 \%$ vs. $73.5 \%$, $P<0.0001$; Fig. 2B). When comparing 5 -year RSR according to age, a relatively young age ( $<50$ years) was associated with better survival compared to those 50 years or older

A

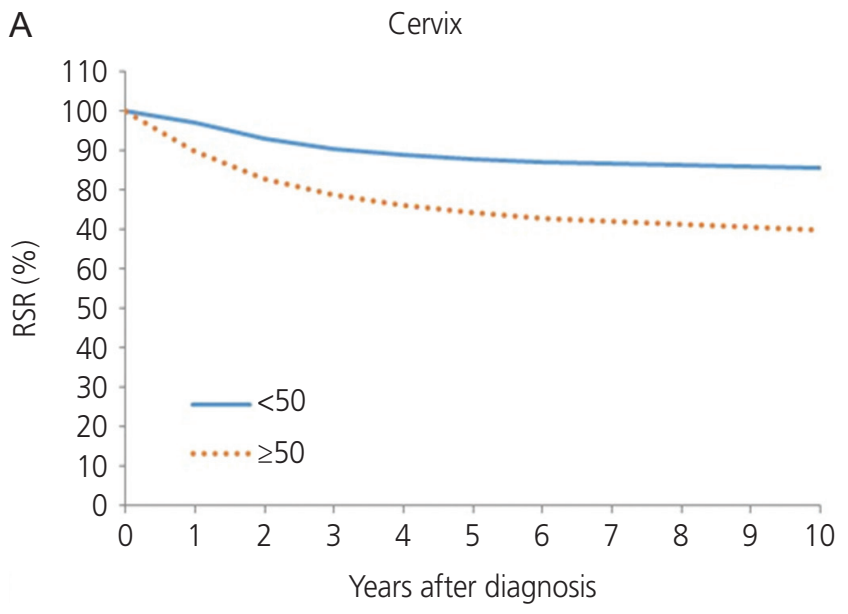

C

Endometrium

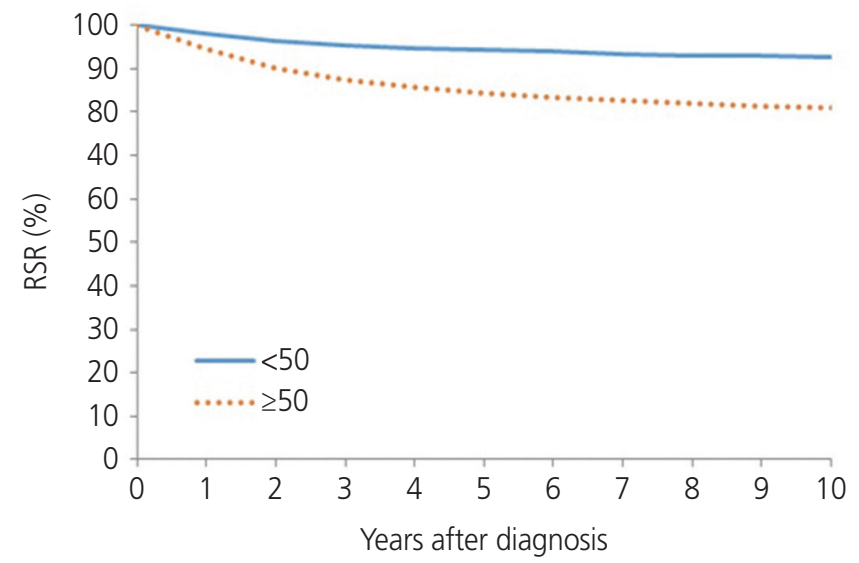

( $87.8 \%$ vs.74.2\%, $P<0.0001$; Fig. $3 \mathrm{~A}$ ). According to the SEER stage, localized, regional, and distant disease showed a 5 -year RSR of $92.5 \%, 72.8 \%$, and $26.9 \%$, respectively $(P<0.0001)$. The unknown stage group showed similar survival to the regional disease group $(72.8 \%$, Fig. 4$)$.

The absolute incidence of POFT cancer in Korea during 1999-2017 increased progressively from 967 in 1999 to 2,505 in 2017. In addition, the ASRs of POFT cancers steadily increased during these years (Table 1). The ASR for POFT cancer increased from 3.7 per 100,000 in 1999 to 6.1 per 100,000 in 2017. The APC was $3.03(95 \% \mathrm{Cl}=2.74$. 3.31; $P<0.001)$ during the observation period. Fig. 1 illustrates the overall APCs for POFT cancers according to age group. The APC of POFT cancers in women younger than 30 years of age was $0.9 \%$, whereas that of POFT cancers in women older than 80 years was $7.0 \%$. The APCs for the $30-39,40-49$, and 50-59 years age groups among females were similar, with an APC of approximately 3. The clinical outcomes of

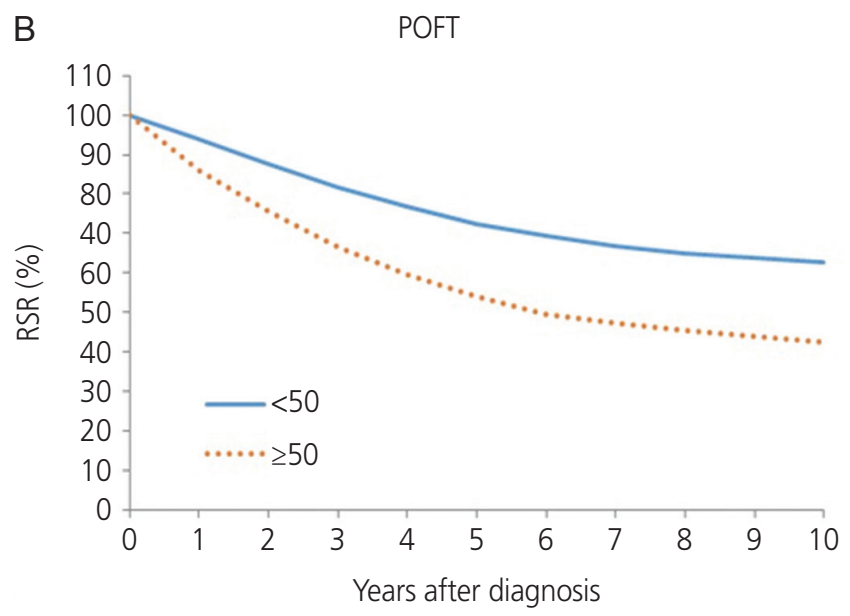

Fig. 3. Relative survival rate (RSR) by cancer type and age group, 1999-2017 (A-C). POFT, primary peritoneal, ovarian epithelial, fallopian tube. 


\section{Obstetrics \& Gynecology Science}

Hyeong In Ha, et al. The incidence and survival of gynecologic cancer in Korea in 1999-2017

POFT cancers were affected by histology, age at diagnosis, and extent of disease. Fig. $2 \mathrm{C}$ depicts the RSR of patients with POFT cancer according to histology. The 5-year RSR of patients with serous POFT cancer was $58.1 \%$. In endometrioid and clear cell histology, the 10-year RSRs were more than $70 \%$ (79.8\% and $75.2 \%$, respectively). Younger patients ( $<50$ years) showed better outcomes than older patients ( $\geq 50$ years) $(72.5 \%$ vs. $54.0 \%$, respectively) (Fig. 3B). Depending on the extent of the disease, localized stage had a better 5-year RSR than regional or distant disease (90.4 vs. 74.6 vs. 42.6 , respectively). For diseases of unknown stage, the survival rate was between that of the regional and distant stages (Fig. 4B).

The annual incidence rate of endometrial cancer increased from 1999 to 2009 (APC, 7.24\%; 95\% Cl=6.29, 8.19). From 2009 to 2014, APC showed a 3.29\% (95\% Cl=0.67, 5.98), and hence the increasing tendency plateaued slightly. From 2014 to 2017, however, the rate of endometrial cancer

A

Cervix

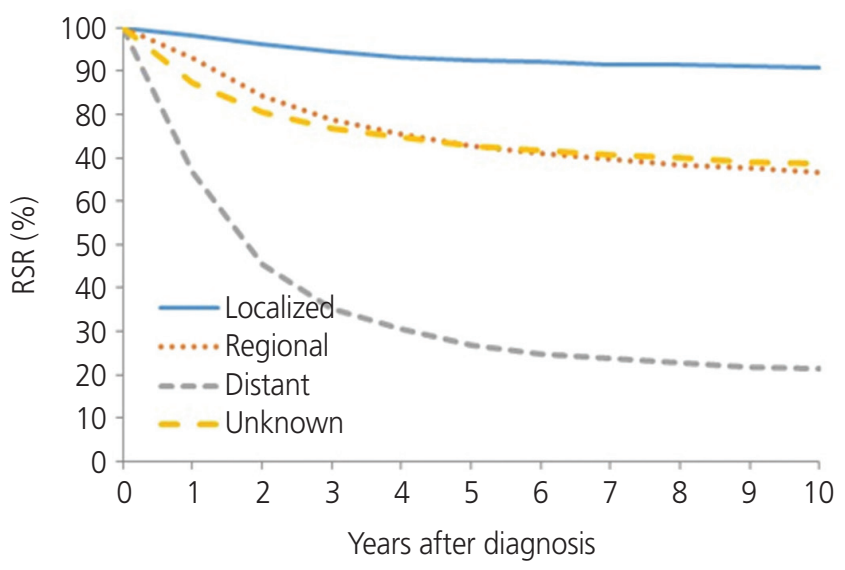

C

Endometrium

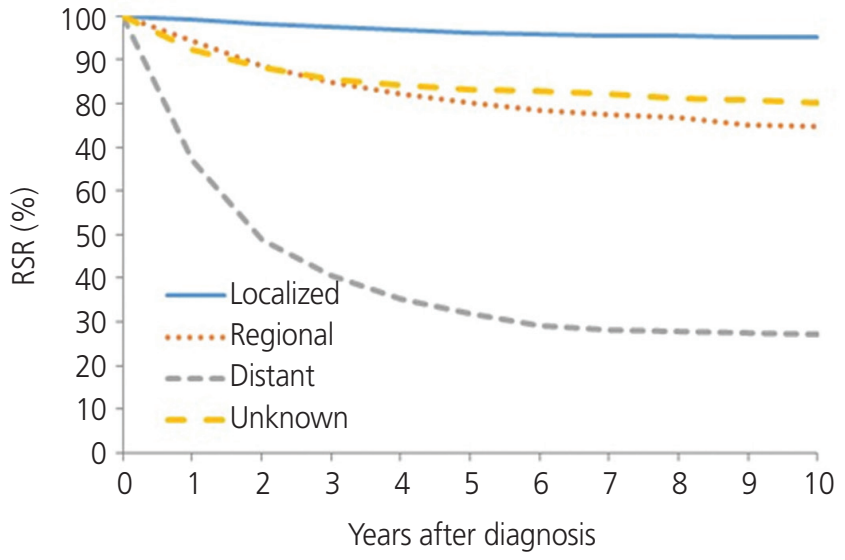

showed a rapid increase (APC, 8.74\%; 95\% Cl=4.99, 12.62) (Table 1). Endometrial cancer showed a positive trend in all age groups. However, younger age groups ( $<30$ and 30-39 years old) and as well as an older age group (60-69 years old) showed the most rapid increasing tendency with regard to the incidence of endometrial cancer (APC, 8.7\%, 7.4\%, and $7.2 \%$, respectively; Fig. 1). The endometrioid histology of endometrial cancer was associated with better survival than non-endometrioid histology in terms of 5-year RSR $(93.2 \%$ vs. $76.5 \%, P<0.0001$; Fig. 2D). When comparing 5 -year RSRs according to age, relatively young age $(<50)$ showed better survival than those aged 50 years or older $(94.3 \%$ vs. $84.4 \%, P<0.0001$; Fig. 3C). According to the SEER stage, localized, regional, and distant disease showed 10-year RSRs of $96.2 \%, 80.3 \%$, and $31.8 \%$, respectively $(P<0.0001)$. The unknown stage group showed similar survival when compared to the regional disease group (Fig. 4C).

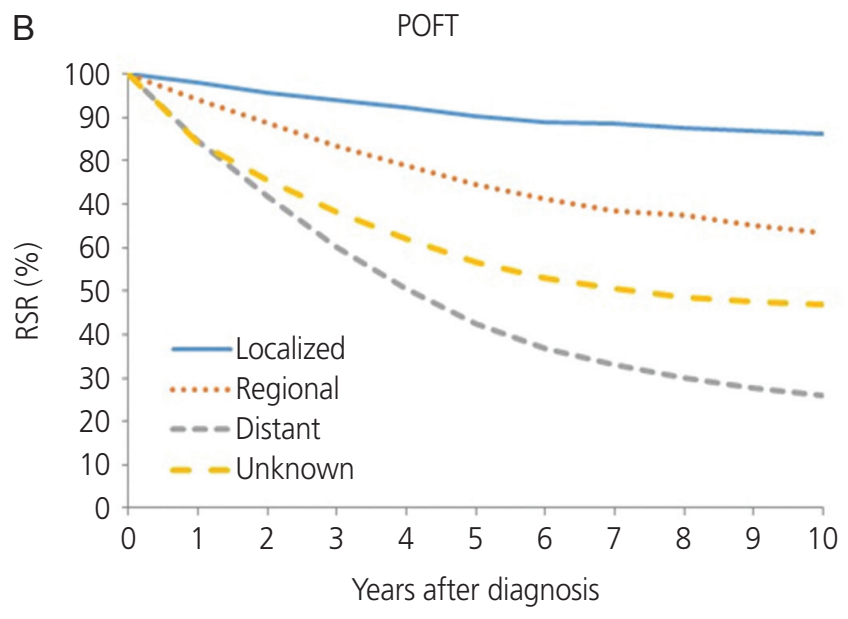

Fig. 4. Relative survival rate (RSR) by cancer type and Surveillance, Epidemiology, and End Results stage, 2006-2017 (A-C). POFT, primary peritoneal, ovarian epithelial, fallopian tube. 


\title{
Obstetrics \& Gynecology Science
}

\author{
Vol. 64, No. 5, 2021
}

\section{Discussion}

In Korea, the incidence of cervical cancer has decreased since the introduction of the National Cervical Cancer Screening program (NCCSP) [10], especially between 1999 and 2017, with an APC of $-4.56 \%$ between 1999 and 2007 and $-2.82 \%$ between 2007 and 2017, respectively. This trend was observed in all age groups, except for those younger than 30 years and older than 80 years.

Human papilloma virus (HPV) prophylactic vaccination has also been introduced into clinical practice since 2007 in Korea, making it possible that this decline in the incidence of cervical cancer more likely to accelerate [11].

Although the incidence of cervical cancer in all women is decreasing (APC, $-4.3 \%$ ), the incidence is increasing in these two subgroups (APC, $4.8 \%$ in women $<30$ years old and $3.2 \%$ in women $\geq 80$ years old). However, it has been suggested that the incidence of cervical cancer among young Korean women has increased during the last two decades [11]. This steady increase in cervical cancer has also been identified in England and is thought to be due to increased exposure to HPV rather than cervical cancer screening [12].

Especially in those younger than 30 years, APC was $1.4 \%$, which is not a large percentage; however, it is important to think of positive APC in young Korean women. Furthermore, changes in sexual activity and the prevalence of HPV infection among Korean women have also been reported [13,14].

During 1999-2017, the incidence of POFT cancers has been increasing progressively. The APC of POFT cancer from 1999 to 2017 was $3.03(95 \% \mathrm{Cl}=2.74$. 3.31, $P<0.001)$ and has been progressively increasing. Because the APC was similar across all sections, there was no need to represent the APC in multiple numbers, unlike cervical cancer or endometrial cancer. Various factors such as hereditary factors, advanced age, nulli-gravity, infertility, hormone replacement therapy, endometriosis, and obesity have been suggested as risk factors for ovarian cancer [15] and are similar to the risk factors for endometrial cancer. However, during the same period, endometrial cancer increased explosively, while ovarian cancer showed a constant increase. Several studies have reported that obesity is related to ovarian cancer development, but the findings of these studies are inconsistent. In addition, there is less increase in risk due to obesity than in endometrial cancer. For the women with a body mass index (BMI) of 35 to 39, the relative risk (RR) of ovarian cancer was 1.51 (CI 1.12-2.02), whereas the RR of endometrial cancer was 2.77 (1.83-4.18) [16]. Even for women with BMI up to 40, the RR was 6.25 (3.75-10.42). These facts seem to have an effect on showing a less steep increase than that of endometrial cancer.

In the present study, the 5-year survival rate of patients with POFT cancer was $61.4 \%$ during 1999-2017. Lee et al. [17] previously reported the 5-year survival rate of epithelial ovarian cancer for 1995-1999 (57.2\%) and 2010-2014 $(63.8 \%)$, before the era of targeted therapy. The 5-year survival rate in the present study seems to be somewhere in between; however, the study contained only epithelial ovarian cancer, and it is impossible to make a direct comparison. Compared to the trends in the 5-year RSRs (\%) by year of diagnosis from 1993 to 2017 in Korea (1993-1995; $60.1 \%, 1996-2000 ; 59.4 \%, 2001-2005 ;$ 61.7\%, 20062010; 61.2\%, 2011-2015; 64.3\%, 2013-2017; 64.9\%) [1]. The present study showed a lower 5-year survival rate, but the report contained not only epithelial ovarian cancer but also germ cell tumors, sex cord stromal tumors, or other malignancies, direct comparisons cannot be made. From the point of survival by histology, endometrioid, mucinous, clear cell carcinoma showed a relatively higher 5 -year survival rate (79.8\%, $76.2 \%$, and $75.2 \%$, respectively) than those with serous histology (58.1\%). Especially in Korea, because clear cell carcinoma histology is increasing [18], it will contribute to an increase in the 5-year survival rate. Therefore, when estimating the cause of improving ovarian cancer survival in Korea, in addition to advances in treatment and surgical methods, changes in histology distribution must be considered.

Increasing endometrial cancer incidence in the young age group might be associated with increased obesity [19], and a recent US population-based cancer registry also showed that young adults showing increasing cancer incidences are associated with obesity, colorectal cancer, and endometrial cancer $[20,21]$. In addition, not only the obesity, but also the metabolic syndrome could be associated with increased incidence of endometrial cancer [22]. A recent meta-analysis showed that parity reduced the risk of endometrial cancer, and decreasing birth rate in Korea maybe associated with an increased rate of endometrial cancer at a young age $[23,24]$. The high prevalence of endometrial cancer in the age group of 60-69 years may be associated with the highest obesity 


\section{Obstetrics \& Gynecology Science}

Hyeong In Ha, et al. The incidence and survival of gynecologic cancer in Korea in 1999-2017

tendency according to the National Health Insurance Service National Sample Cohort from 2012 to 2013 [25].

Although it is known that there is a mechanism in which type 1 endometrial cancer is hormone-dependent, a previous report has shown that there is no difference in age at diagnosis between type 1 and type 2 [26]. This can be interpreted that in our study results, the incidence rate of intimal cancer increases with age and may not be related to endometrial cancer type. The decrease in the birth rate in Korea and the increase in unmarried women may increase the incidence of endometrial cancer in the young population [23].

In this study, the 5-year RSR by SEER stage was $94.2 \%$, $80.3 \%$, and $31.8 \%$ for localized, regional, and distant stages, respectively. Compared to a previous report based on the Korea National Cancer Incidence Database from 1999 to 2017 , the 5-year RSR of endometrial cancer was similar $(95.7 \%, 81.4 \%$, and $32.6 \%)$ in our study [1].

The survival outcome of endometrial cancer in the unknown stage was worse than that in the localized stage and a better survival rate than the regional and distant stages. The unknown stage group might have included occult endometrial cancer. Occult endometrial cancer has been reported to have $0.2-0.75 \%$ chance of occurring after hysterectomy $[27,28]$. In presumed stage I disease, lymphadenectomy may not decrease death or disease recurrence risk, as shown in the Cochrane review [29]. However, there may have been lymph node metastasis that was not detected on preoperative imaging, and this may have lowered the survival rate of the unknown group compared to localized disease due to the omission of adjuvant treatment. Lymph node metastasis may have not been detected on preoperative imaging, and this may lower the survival rate compared to localized disease due to the omission of adjuvant treatment.

The incidence of gynecologic cancer in Korea and changes in clinical outcomes were investigated in the present study. Gynecologic oncologists should make efforts to prevent and treat malignancy in line with the change in cancer etiology. In particular, the importance of primary prevention should not be ignored. Cervical cancer is gradually decreasing due to the steady establishment of a national screening program, improvement of hygiene conditions, and dissemination of vaccinations. Therefore, we continue to implement the current screening program well, inform the public about vaccination against HPV, and push for improvements in national medical policies. In addition, the risk of endometrial cancer is increasing due to westernized society and increased obesity rate. When patients visit the hospital, they are educated not only about their current disease but also about weight and lifestyle management. Moreover, regular gynecological examinations are recommended. In the case of POFT cancers, to date, major clinical trials have generally shown little or no benefit with screening using ultrasound and CA125. While much research has been undertaken to find potential new biomarkers, POFT cancer screening has not been improved. Therefore, there is a need for continuous research on methods to detect POFT cancer at an early stage, and at the same time, the focus should be placed on high-risk groups for ovarian cancer. Patients with a strong family history of cancer, such as breast, ovarian, and colorectal cancers, should be guided to visit genetic counseling clinics. A family tree and familial genetic test should be performed, and if genetic variants, especially BRCA1 and BRCA2 variants, are found, sufficient information should be provided for aggressive surgery such as RRSO or prophylactic mastectomy.

The incidence rates for gynecologic cancers decreased by approximately 1.76 per year until 2005, followed by nonsignificant changes until 2017, and increased thereafter with an annual increase of 2.76 until 2017. The highest 5-year survival rates were observed for endometrial and cervical cancers. The younger the age and the narrower the disease range, the better the survival outcome.

\section{Conflict of interest}

No potential conflict of interest relevant to this article was reported.

\section{Ethical approval}

This study was approved by the Institutional Review Board of the National Cancer Center, Korea (NCC2020-0176). The study was performed in accordance with the principles of the Declaration of Helsinki.

\section{Patient consent}

Written informed consent and the use of images from pa- 


\section{Obstetrics \& Gynecology Science}

Vol. 64, No. 5, 2021

tients are not required for the publication.

\section{Funding information}

None.

\section{Supplementary material}

Supplementary Table 1-4 associated with this article can be found online at https://doi.org/10.5468/ogs.21116.

\section{References}

1. Hong S, Won YJ, Park YR, Jung KW, Kong HJ, Lee ES. Cancer statistics in Korea: incidence, mortality, survival, and prevalence in 2017. Cancer Res Treat 2020;52:33550.

2. Shin HR, Won YJ, Jung KW, Kong HJ, Yim SH, Lee JK, et al. Nationwide cancer incidence in Korea, 1999 2001; first result using the national cancer incidence database. Cancer Res Treat 2005;37:325-31.

3. Chung HH, Jang MJ, Jung KW, Won YJ, Shin HR, Kim JW, et al. Cervical cancer incidence and survival in Korea: 1993-2002. Int J Gynecol Cancer 2006;16:1833-8.

4. Chung HH, Hwang SY, Jung KW, Won YJ, Shin HR, Kim JW, et al. Ovarian cancer incidence and survival in Korea: 1993-2002. Int J Gynecol Cancer 2007;17:595-600.

5. Jung KW, Won YJ, Kong HJ, Lee ES. Cancer statistics in Korea: incidence, mortality, survival, and prevalence in 2016. Cancer Res Treat 2019;51:417-30.

6. World Health Organization. International statistical classification of diseases and related health problems. 10th ed. Geneva: World Health Organization Press; 1994.

7. Segi M, Fujisaku S. Cancer mortality for selected sites in 24 countries (1950-1957). Sendai: Tohoku University School of Medicine; 1960.

8. National Cancer Institute. SEER Summary staging manual 2000: codes and coding instructions. Bethesda: National Cancer Institute; 2000.

9. Ederer $F$, Heise $\mathrm{H}$. Instructions to IBM 650 programmers in processing survival computations. Bethesda: National Cancer Institute; 1959.
10. Oh CM, Jung KW, Won YJ, Shin A, Kong HJ, Jun JK, et al. Trends in the incidence of in situ and invasive cervical cancer by age group and histological type in Korea from 1993 to 2009. PLoS One 2013;8:e72012.

11. Lim MC, Moon EK, Shin A, Jung KW, Won YJ, Seo SS, et al. Incidence of cervical, endometrial, and ovarian cancer in Korea, 1999-2010. J Gynecol Oncol 2013;24:298302.

12. Patel A, Galaal K, Burnley C, Faulkner K, Martin-Hirsch $\mathrm{P}$, Bland $\mathrm{MJ}$, et al. Cervical cancer incidence in young women: a historical and geographic controlled UK regional population study. Br J Cancer 2012;106:1753-9.

13. Kim CJ, Kim BG, Kim SC, Kim YT, Kim YM, Park SY, et al. Sexual behavior of Korean young women: preliminary study for the introducing of HPV prophylactic vaccine. Korean J Gynecol Oncol 2007;18:209-18.

14. Kim YT. Current status of cervical cancer and HPV infection in Korea. J Gynecol Oncol 2009;20:1-7.

15. Hunn J, Rodriguez GC. Ovarian cancer: etiology, risk factors, and epidemiology. Clin Obstet Gynecol 2012;55:323.

16. Calle EE, Rodriguez C, Walker-Thurmond K, Thun MJ. Overweight, obesity, and mortality from cancer in a prospectively studied cohort of U.S. adults. N Engl J Med 2003;348:1625-38.

17. Lee JY, Kim S, Kim YT, Lim MC, Lee B, Jung KW, et al. Changes in ovarian cancer survival during the 20 years before the era of targeted therapy. BMC Cancer 2018;18:601.

18. Kim SI, Lim MC, Lim J, Won YJ, Seo SS, Kang S, et al. Incidence of epithelial ovarian cancer according to histologic subtypes in Korea, 1999 to 2012. J Gynecol Oncol 2016;27:e5.

19. Baik I. Forecasting obesity prevalence in Korean adults for the years 2020 and 2030 by the analysis of contributing factors. Nutr Res Pract 2018;12:251-7.

20. Wise MR, Jordan V, Lagas A, Showell M, Wong N, Lensen $S$, et al. Obesity and endometrial hyperplasia and cancer in premenopausal women: a systematic review. Am J Obstet Gynecol 2016;214:689.e1-17.

21. Sung $H$, Siegel RL, Rosenberg PS, Jemal A. Emerging cancer trends among young adults in the USA: analysis of a population-based cancer registry. Lancet Public Health 2019;4:e137-47.

22. Lee DY, Lee TS. Associations between metabolic syn- 


\section{Obstetrics \& Gynecology Science}

Hyeong In Ha, et al. The incidence and survival of gynecologic cancer in Korea in 1999-2017

drome and gynecologic cancer. Obstet Gynecol Sci 2020;63:215-24.

23. Song JE, Ahn JA, Lee SK, Roh EH. Factors related to low birth rate among married women in Korea. PLoS One 2018;13:e0194597.

24. Raglan O, Kalliala I, Markozannes G, Cividini S, Gunter MJ, Nautiyal J, et al. Risk factors for endometrial cancer: an umbrella review of the literature. Int J Cancer 2019;145:1719-30.

25. Park HJ, Hong YH, Cho YJ, Lee JE, Yun JM, Kwon H, et al. Trends and cut-point changes in obesity parameters by age groups considering metabolic syndrome. J Korean Med Sci 2018;33:e47.

26. Bjørge T, Engeland A, Tretli S, Weiderpass E. Body size in relation to cancer of the uterine corpus in 1 million Norwegian women. Int J Cancer 2007;120:378-83.

27. Parsons LHP, Pedersen R, Richardson DL, Kho KA. The prevalence of occult endometrial cancer in women undergoing hysterectomy for benign indications. Eur J Obstet Gynecol Reprod Biol 2018;223:108-12.

28. Desai VB, Wright JD, Gross CP, Lin H, Boscoe FP, Hutchison LM, et al. Prevalence, characteristics, and risk factors of occult uterine cancer in presumed benign hysterectomy. Am J Obstet Gynecol 2019;221:39.e1-14.

29. Frost JA, Webster KE, Bryant A, Morrison J. Lymphadenectomy for the management of endometrial cancer. Cochrane Database Syst Rev 2017;10:CD007585. 